\title{
Utility of stone volume estimated by software algorithm in predicting success of medical expulsive therapy
}

Rajat Jain, MD ${ }^{1}$; Sara Maskal, BS ${ }^{2}$; Jason Milk, MD³; Leonard Kahn, MD ${ }^{4}$; Donald Fedrigon III, $\mathrm{BS}^{2}$; Sri Sivalingam, $\mathrm{MD}^{5}$

${ }^{1}$ University of Rochester School of Medicine and Dentistry, Rochester, NY, United States; ${ }^{2}$ Case Western Reserve School of Medicine, Cleveland, OH, United States; ${ }^{3}$ Cleveland Clinic Department of Emergency Medicine, Cleveland, $\mathrm{OH}$, United States; ${ }^{4}$ Cleveland Clinic Glickman Department of Radiology, Cleveland, OH, United States; ${ }^{5}$ Cleveland Clinic Glickman Urological and Kidney Institute, Cleveland, OH, United States

Cite as: Jain R, Maskal S, Milk J, et al. Utility of stone volume estimated by software algorithm in predicting success of medical expulsive therapy. Can Urol Assoc J 2020 August 7; Epub ahead of print. http://dx.doi.org/10.5489/cuaj.6491

Published online August 7, 2020

$* * *$

\section{Abstract}

Introduction: We sought to assess the accuracy of using stone volume (SV) estimated with a software algorithm as a predictor for stone passage in a trial of medical expulsive therapy (MET).

Methods: We identified patients with ureteral stones discharged from the ER on MET. Patients with infection, non-ureteral stones, or needing immediate surgical intervention were excluded. For each stone, longest dimension (LD) was recorded and SV was estimated by a computed tomography (CT)-based region growing (RG) algorithm and standard ellipsoid formula (EF). Stone passage within 30 days was assessed via electronic chart and followup phone call.

Results: Fifty-one patients were included for analysis ( $53 \pm 16.7$ years, $24 \%$ female). The mean LD was $4.85 \pm 2.02 \mathrm{~mm}$. The mean SV was similar by EF and RG $\left(0.051 \pm 0.057 \mathrm{~cm}^{3}\right.$ vs. $0.049 \pm$ $\left.0.052 \mathrm{~cm}^{3} ; \mathrm{p}=0.28\right)$. Thirty-three $(65 \%)$ patients passed their stone, while $18(35 \%)$ did not. The mean LD for passed stones vs. failed passage was $4.1 \pm 1.7 \mathrm{~mm}$ vs. $6.2 \pm 1.8 \mathrm{~mm}(\mathrm{p}=0.0002)$; the mean EF volume was $0.028 \pm 0.035 \mathrm{~cm}^{3}$ vs. $0.093 \pm 0.066 \mathrm{~cm}^{3}(\mathrm{p}=0.00007)$; and the mean volume by RG was $0.028 \pm 0.027 \mathrm{~cm}^{3}$ vs. $0.088 \pm 0.063 \mathrm{~cm}^{3}$ ( $\left.\mathrm{p}=0.00005\right)$.

Conclusions: The clinical utility of using SV estimated by software algorithm as a predictor for success of MET has not previously been examined. We demonstrate that spontaneously passed stones had a significantly smaller volume than those requiring intervention. Further prospective studies are needed to validate these findings and establish volume thresholds for probability of stone passage. 


\section{Introduction}

In patients presenting to the emergency room (ER) with ureteral stones, accurate assessment of stone burden is important in evaluation and management. Both treatment selection and prediction of successful outcome are dependent on accurate assessment of stone burden.

The gold-standard imaging modality in the urolithiasis patient is non-contrast computed tomography (CT) of the abdomen and pelvis. ${ }^{1,2}$ Using CT provides information regarding size, shape, density, and location of stones. Traditionally, the longest dimension (LD) on CT is used as a surrogate for stone burden. Previously, LD was used to predict stone passage successfully in $88 \%$ of cases. ${ }^{3}$ Kidney stones are three dimensional, often irregular in shape, and orientation may not correspond with LD being in the axial plane. As such, using LD is a suboptimal surrogate for stone volume and burden. Further, there can be significant variation in manually performed linear measurements on CT images. ${ }^{4}$

Stone volume (SV) may be an important predictor of outcome in treatment of stone disease. ${ }^{5,6}$ We have previously shown that an automated CT-based region-growing (RG) algorithm accurately and reliably estimated stone volumes in an in vitro study. ${ }^{7}$ To our knowledge, stone volume estimated by such software algorithms has not been previously examined in a clinical context. We hypothesize that SV is a better measurement for prediction of spontaneous stone passage in patients given trial of medical expulsive therapy (MET).

\section{Methods}

Consecutive patients presenting to the ER with a single ureteral stone and discharged on medical expulsive therapy (MET) were identified by ER physicians between March 2017 and January 2018. Medical Expulsive Therapy at our institution consists of 14-21 days of Tamsulosin and guidance to stay hydrated and use NSAIDs for pain. Patients were also prescribed additional analgesia (typically toradol or tramadol) and colace per provider discretion. All patients included in this study were discharged with MET. Patients who had urinalysis suggestive of infection, non-ureteral stones, and those who required immediate surgical intervention due to intractable pain, vomiting or acute kidney injury were excluded. All patients underwent standard stoneprotocol CT of the abdomen and pelvis without contrast. The CT scanner provides isotropic resolution of $1 \mathrm{~mm}$ in all three planes, which is standard for our institution. One millimeter cuts are important for more sophisticated analysis of the images by the radiologist as well as more accurate volume assessment. Under normal circumstances, after the imaging study has been analyzed and read, only every third cut is saved to the PACS (picture archiving and communication system) for archiving to reduce storage requirements. For this collaborative study, radiology staff were notified to save all imaging information from the original study for each patient identified in the emergency room. Imaging studies with $1 \mathrm{~mm}$ cuts were imported in the electronic medical record (EMR). Patients for whom $1 \mathrm{~mm}$ cuts could not be saved for further analysis were also excluded. A radiologist used the AGFA IMPAX Volume Viewing 3D 
software to estimate volume for all stones included by region growing algorithm (RG) and ellipsoid formula (EF) as previously described. ${ }^{7}$ We also recorded the LD (in axial, coronal or sagittal plane) for each stone. We assessed whether the patient spontaneously passed the stone within 30 days or required surgical intervention by examining the electronic medical record and via patient phone call. Thus we were able to get a true stone passage rate. Patient demographics and clinical characteristics were recorded.

Stone volumes were stratified into quartiles for each measurement. Comparisons between groups were done using Wilcoxon rank sum test for continuous variables and chi-square test or Fisher's exact test for binary or categorical variables. Logistic regression analysis was applied to assess passing probability for stone size estimated by each of the 3 methods. Multivariable logistic regression also was used to estimate the effect of patient age and gender. Odds ratio and its $95 \%$ CI of stone passing was estimated for each variable examined. Results were considered significant at the $\mathrm{p}=0.05$ level. The statistical software used is $\mathrm{R}$ (version 3.3.2: R Core Team (2016). R: A language and environment for statistical computing. R Foundation for Statistical Computing, Vienna, Austria. URL: https://www.R-project.org).

\section{Results}

We identified 90 patients with ureteral stones who were discharged on MET. Twenty eight patients were excluded because $1 \mathrm{~mm}$ cuts were not saved for review; 3 patients were excluded because stones suspected to be at the ureterovesical junction were confirmed to be in the bladder on review by the radiologist; 8 patients were excluded due to inability to follow up with the patient for confirmation of outcome following ER discharge.

Fifty one patients were ultimately included in the analysis (Table 1). Mean age was $53 \pm$ 16.7 years. Twenty four percent of patients were female, while the remainder were male. The mean LD was $4.85 \pm 2.02 \mathrm{~mm}$. The mean stone volume estimated by EF was $0.051 \pm 0.057 \mathrm{~cm}^{3}$ while the mean stone volume estimated by RG was $0.049 \pm 0.052 \mathrm{~cm} 3$. There was no statistically significant difference between the two estimates $(\mathrm{p}=0.28)$.

For all three measures, stones were stratified into quartiles. Figure 1 shows the distribution of the average value of each quartile.

Thirty three (65\%) patients passed their stone while $18(35 \%)$ did not (Table 1). Age was similar between the two groups ( 58.5 vs 50.9 years, $\mathrm{p}=0.13)$. Gender distribution was also similar (percent female: $18 \%$ vs $33 \%, \mathrm{p}=0.22$ ). The mean LD for passed stones was $4.1 \pm 1.7 \mathrm{~mm}$ compared to $6.2 \pm 1.8 \mathrm{~mm}(\mathrm{p}=0.0002)$. The mean EF volume was $0.028 \pm 0.035 \mathrm{~cm}^{3}$ versus 0.093 $\pm 0.066 \mathrm{~cm}^{3}(\mathrm{p}=0.00007)$. The mean volume by $R G$ was $0.028 \pm 0.027 \mathrm{~cm}^{3}$ versus $0.088 \pm$ $0.063 \mathrm{~cm}^{3}(\mathrm{p}=0.00005)$.

Age and gender were not significantly associated with passage of stones on univariate analysis ( $p=0.1083$ and $p=0.2286$, respectively). All three measures, $L D, R G$, and $E F$, were independently associated with passage of stones $(\mathrm{LD}$ OR $=0.19, \mathrm{p}=0.0012$; $\mathrm{RG}$ OR $=0.16, \mathrm{p}=$ 
0.0016; $\mathrm{EF} \mathrm{OR}=0.24, \mathrm{p}=0.0019$, respectively). All three had similar C statistics $(0.818,0.846$ and 0.839 , respectively). However, $95 \%$ confidence intervals for the odds ratio overlapped, indicating no difference in predictive power between the three measures.

\section{Discussion}

Currently, there is some debate regarding the efficacy of MET. ${ }^{8-12}$ We examined whether using a novel metric, stone volume, could give clinicians an additional data point in clinical decision making in MET. Stone volume as a measure of stone burden has been shown to be predictive of treatment outcome..$^{5,6}$

Current guidelines suggest using LD as a surrogate for stone burden in clinical decisionmaking in patients presenting with urolithiasis. ${ }^{2,13}$ However, LD is a relatively poor surrogate given the three dimensional nature of stones. There are several reasons for this. First, reconstructed images in the coronal and sagittal planes can be overlooked in assessing the true LD of a ureteral stone. Second, as the stone size increases and shape gets more irregular, a single LD measurement represents the true clinical implications of the stone even more poorly. Third, Patel et al. have shown significant variability in manual linear measurements compared to automated measurements on CT studies, making LD less reliable than an automated estimate. ${ }^{4}$ Lastly, individual software settings preferred by the reader and window parameters significantly affect the variability in measurement. ${ }^{4}$

In a previous in vitro study, we demonstrated that the region-growing algorithm is accurate and reliable when estimating true volume by water displacement. ${ }^{7}$ In the present study we applied SV estimated using the region growing algorithm in a clinical context. Patients with ureteral stones who were discharged from the ER on MET were followed for one month to assess rate of stone passage. Stone volumes were estimated using the region-growing algorithm and the ellipsoid formula.

We found that the all three measures, LD, EF and RG, had a statistically significant relationship to stone passage rate. Stones that passed were significantly smaller by all three measures than those that did not pass. Further, all three measures showed decreased odds of passing stones with increasing LD or volume on logistic regression. The volume estimates for $\mathrm{RG}$ and $\mathrm{EF}$ were internally consistent at every quartile. In contrast, there were stark differences between LD and the other methods at the lowest and highest quartiles. LD estimated volume was significantly greater than estimates due to RG or EF in the first quartile, but significantly lower than RG and EF estimates in the fourth quartile. We felt that these differences were likely due to the major intrinsic limitation of the LD method: only a single radius measurement was available for LD calculations, but this does not account for the non-uniform shapes of stones. The $\mathrm{C}$ statistic, and therefore, the predictive power, on each estimate was not significantly different. Although statistical significance was not found in our study, the data showed a trend towards greater consistency in the volume-based methods of calculation. 
Zorba et al have previously shown that there is significant difference between stone volume in spontaneously passed stones versus those that did not pass. ${ }^{5}$ This study used the ellipsoid formula for SV estimation. In the present study, we showed that stone volume thresholds based on RG algorithm and EF estimates are both associated with stone passage.

We had previously shown in an in vitro model that RG is an overall more accurate and reliable estimate of stone volume than EF for medium and larger sized stones. However, for smaller stones, as in those included in this study, they were comparable. ${ }^{7}$ Thus, one may use either technique to obtain relatively accurate stone volumes in this clinical scenario. However, based on our own results and extrapolation from Patel et al, EF might be a significantly more variable estimate due to inter-observer measurement variance. ${ }^{4}$

As a technique in its infancy, stone volume calculation must undergo significant validation and standardization before it can be used to routine clinical decision making. Advancements in volume measurement with reduced radiation doses are being made which will contribute to its adoption. ${ }^{14}$ As CT software becomes more sophisticated and automated, additional metrics such as volume may eventually become standard, similar to the use of skin to stone distance or Hounsfield unit density.

This study involved significant collaboration between the urology, radiology and emergency departments. This represents a consecutive cohort of patients that matched the inclusion criteria as identified by emergency room staff. Further, due to our rigorous follow up via phone call, we were able to calculate the true stone passage rate in our patient population. By doing this, we were able to avoid the limitation of some MET studies where true stone passage rate is not known, or surrogate metrics are used to assume stone passage.

Limitations of this study include a relatively small cohort, as well as a significant percentage of patients who were excluded due to incomplete raw image data for analysis. It is unlikely that a selection bias resulted from this, however, as this was due to technical and software limitations rather than any clinical metric. A larger training cohort would improve the ability to predict likelihood of stone passage within different volume ranges. Further, external prospective validation of our findings would be critical to building a case for use of stone volume in routine clinical decision making.

\section{Conclusions}

The clinical utility of using stone volume estimated by a software algorithm as a predictor for success of MET has not previously been examined. We demonstrate that the volume of stones that passed spontaneously was significantly smaller than for those that required intervention. Although the algorithmic volume estimate was not more predictive of stone passage than ellipsoid formula or longest dimension, it has the potential to be an improvement over other metrics because it is less operator dependent and more consistent between trials. Further 
prospective studies are needed to validate these findings and establish potential volume thresholds for probability of spontaneous passage.

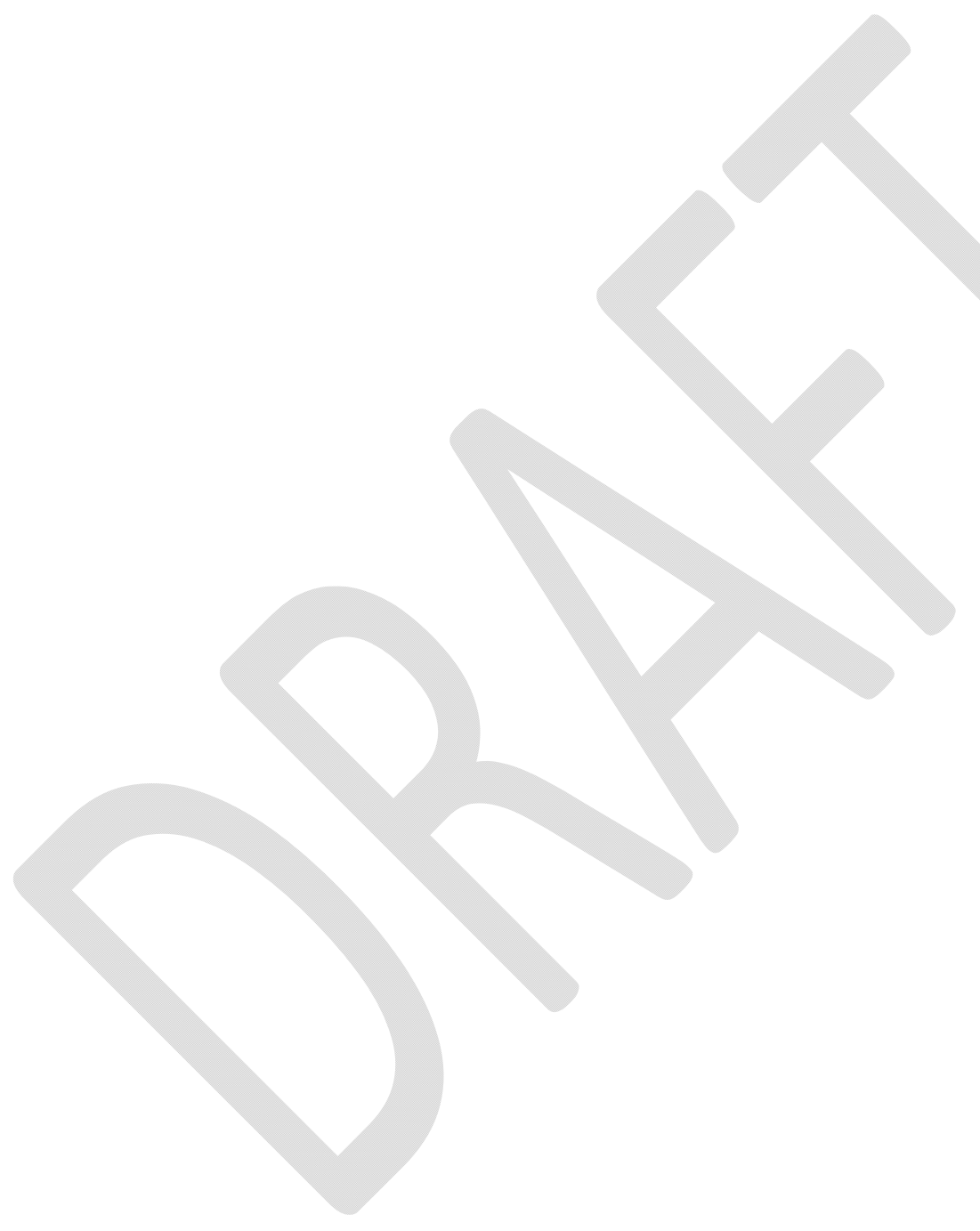




\section{References}

1. Niemann T, Kollmann T, Bongartz G. Diagnostic performance of low-dose CT for the detection of urolithiasis: A meta-analysis. Am J Roentgenol. 2008;191(2):396-401. doi:10.2214/AJR.07.3414.

2. Türk C, Petř́k A, Sarica K, et al. EAU Guidelines on Diagnosis and Conservative Management of Urolithiasis. Eur Urol. 2015;69:1-7. doi:10.1016/j.eururo.2015.07.040.

3. Parekattil SJ, Kumar U, Hegarty NJ, et al. External Validation of Outcome Prediction Model for Ureteral/Renal Calculi. J Urol. 2006;175(2):575-579. doi:10.1016/S00225347(05)00244-2.

4. Patel SR, Wells S, Ruma J, et al. Automated volumetric assessment by noncontrast computed tomography in the surveillance of nephrolithiasis. Urology. 2012;80(1):27-31. doi:10.1016/j.urology.2012.03.009.

5. Zorba OU, Ogullar S, Yazar S, Akca G. Ct-Based Determination of Ureteral Stone Volume: a Predictor of Spontaneous Passage. J Endourol. 2015;30(1):32-36. doi:10.1089/end.2015.0481.

6. Bandi G, Meiners RJ, Pickhardt PJ, Nakada SY. Stone measurement by volumetric threedimensional computed tomography for predicting the outcome after extracorporeal shock wave lithotripsy. BJU Int. 2009;103(4):524-528. doi:10.1111/j.1464-410X.2008.08069.x.

7. Jain R, Omar M, Chaparala H, et al. How accurate are we in measuring stone volume? A comparison of water displacement, ellipsoid formula, and a CT-based software tool. $J$ Endourol. 2018.

8. Pickard R, Starr K, MacLennan G, et al. Medical expulsive therapy in adults with ureteric colic: a multicentre, randomised, placebo-controlled trial. Lancet. 2015;386(9991):341349. doi:10.1016/S0140-6736(15)60933-3.

9. Furyk JS, Chu K, Banks C, et al. Distal Ureteric Stones and Tamsulosin: A DoubleBlind, Placebo-Controlled, Randomized, Multicenter Trial. Ann Emerg Med. 2016;67(1):86-95.e2. doi:10.1016/J.ANNEMERGMED.2015.06.001.

10. Arda E, Cakiroglu B, Yuksel I, Akdeniz E, Cetin G. Medical Expulsive Therapy for Distal Ureteral Stones: Tamsulosin Versus Silodosin in the Turkish Population. Cureus. 2017;9(11):e1848. doi:10.7759/cureus.1848.

11. Ye Z, Zeng G, Yang H, et al. Efficacy and Safety of Tamsulosin in Medical Expulsive Therapy for Distal Ureteral Stones with Renal Colic: A Multicenter, Randomized, Double-blind, Placebo-controlled Trial. Eur Urol. November 2017. doi:10.1016/J.EURURO.2017.10.033.

12. Hollingsworth JM, Canales BK, Rogers MAM, et al. Alpha blockers for treatment of ureteric stones: systematic review and meta-analysis. BMJ. 2016;355:i6112. doi:10.1136/BMJ.I6112.

13. Assimos D, Krambeck A, Miller NL, et al. Surgical Management of Stones: American Urological Association/Endourological Society Guideline, PART II. Vol 196.; 2016. doi:10.1016/j.juro.2016.05.091.

14. Takahashi S, Itoh $\mathrm{T}$, Niikawa $\mathrm{H}$, et al. Two-pass dual-energy $\mathrm{CT}$ imaging for simultaneous detection, characterization, and volume measurement of urinary stones with 
excretory-phase CT urography alone: a phantom study. Jpn J Radiol. 2013;31(6):393400. doi:10.1007/s1 1604-013-0209-5. 


\section{Figures and Tables}

Fig. 1. Distribution of all three measures plotted by averages of quartiles. EF: ellipsoid formula; LD: longest dimension; RG: region growing.

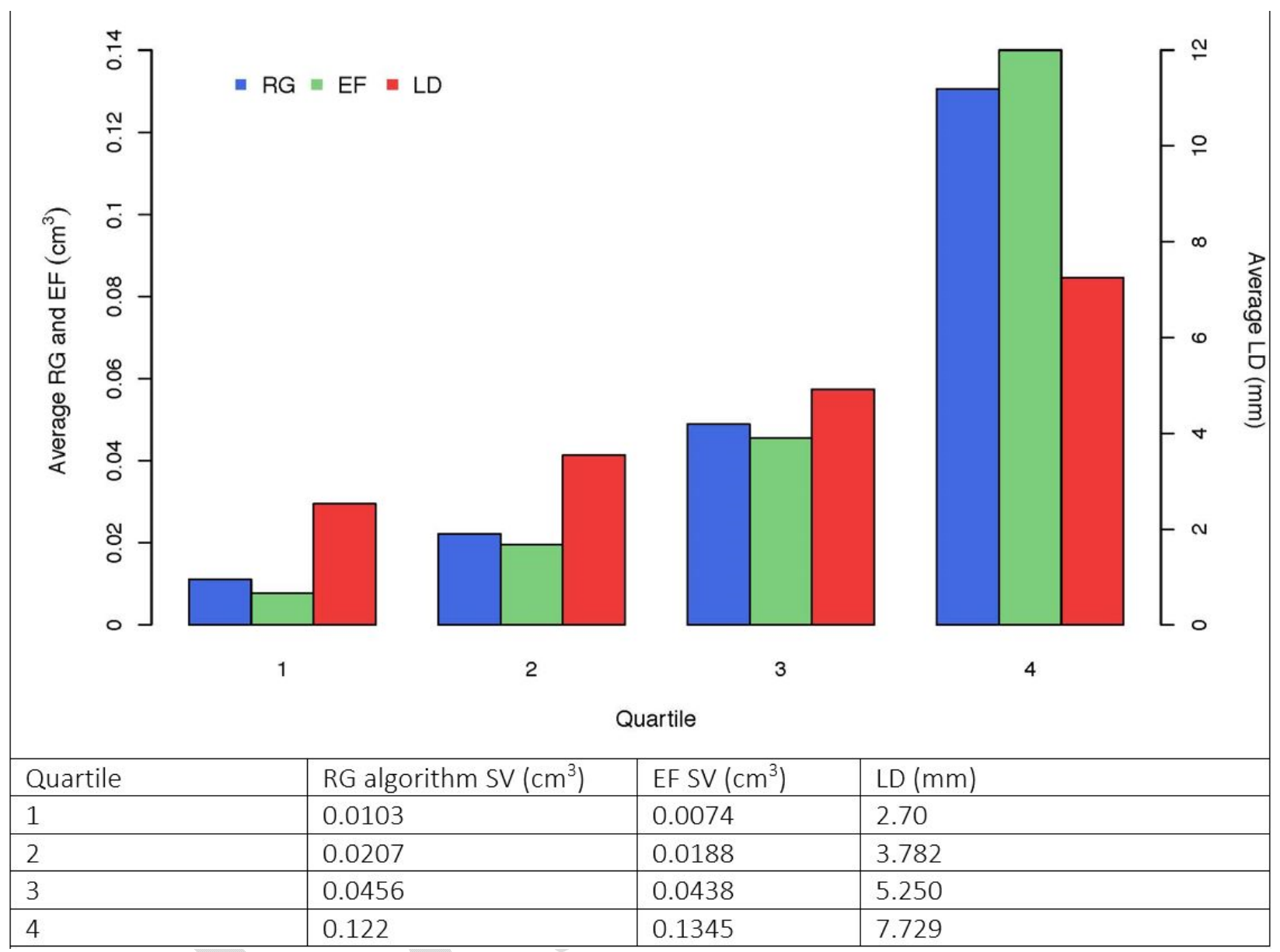




\begin{tabular}{l} 
Table 1. Comparison of characteristics between stones that passed versus those requiring \\
intervention \\
\hline
\end{tabular}

EF: ellipsoid formula; LD: longest dimension; RG: region growing. 